\title{
Factors affecting decision concerning influenza vaccination among students of medical faculties
}

\author{
Agnieszka Woźniak-Kosek ${ }^{1 凶}$, Bogumiła Kempińska-Mirosławska², Mariola Mendrycka³, \\ Agnieszka Saracen ${ }^{4}$ and Grażyna Hoser ${ }^{1}$
}

'Centre of Postgraduate Medical Education, Laboratory of Flow Cytometry, Warsaw, Poland; ${ }^{2}$ Department of History of Medicine, Pharmacy and Military Medicine at the Medical University of Łódź, Łódź, Polad; ${ }^{3}$ Department of Material Science, Technology and Design, Department of Chemistry and Polymer Technology; University of Technology and Life Sciences in Radom, Radom, Poland; “Department of Health and Physical Education, University of Technology and Life Sciences in Radom, Radom, Poland

Influenza is one of the most common cyclic respiratory diseases in humans. Methods of prevention are multidirectional, but the most effective and most efficacious way to prevent influenza and its complications is through preventive vaccination. This work aims to determine different factors affecting the decision concerning influenza vaccine. The percentage of people vaccinated against the flu was evaluated, as well as their knowledge of post-influenza complications, etc. among full-time students and bridging studies of nursing and physiotherapy (full-time and part-time) at the University of Technology and Life Sciences in Radom, and students of medicine and pharmacy at the Medical University of Łódź. The research tool was the authors' questionnaire with 18 questions. The surveys conducted, consisting of multiple choice questions, were anonymous. In total, the survey involved 470 students. Overall, the number of people who were vaccinated against influenza in the 2012/13 epidemic season numbered 15 respondents, representing $5.84 \%$ of the total group of respondents. For the group of nursing students it was $6 \%$, for physiotherapy students $5 \%$, for students of medicine and pharmacy $14 \%$. The percentage of respondents who said they would get vaccinated if the vaccinaton was free of charge was also low. Increasing the percentage of people vaccinated against influenza (immunization coverage) is a very important measure in preventing influenza epidemics. Therefore, it is necessary to identify the reasons why people are reluctant to be vaccinated against influenza, particularly among students who will work in the future in the health care services sector.

Key words: influenza, vaccination against influenza- knowledge, infections, students

Received: 23 January, 2014; revised: 29 September, 2014; accepted: 10 December, 2014; available on-line: 19 December, 2014

\section{INTRODUCTION}

Influenza is an important clinical, epidemiological, and economic problem. It is one of the most common infectious viral diseases and a significant cause of morbidity and mortality in many regions of the world. According to estimates of the World Health Organization (WHO), every year 330 million to 1.575 billion people get influenza or diseases with influenza-like symptoms, where 500 thousand to a million of them die (Brydak, 2008). In the last epidemic season in Poland there were over 2.7 million cases or suspected cases of the flu, more than 12000 influenza-related hospitalisations, and 119 deaths (The Reports NIPH-NIH, 2013). In relation to low influenza vaccination coverage $3.7 \%$ of the population in the 2012/2013 epidemic season) it is necessary to take measures for the promotion of knowledge about the dangers of influenza, its complications, and their economic and social impact. A special role in this regard should be played by medical personnel, from whom not only professionalism is expected, but also appropriate attitudes and behaviours in terms of their own and patients' health (Maszke et al., 2013; Woźniak-Kosek et al., 2014).

In the prevention of influenza, isolation procedures and appropriate hygiene behaviour play important roles, but pharmacological prophylaxis is also possible. The main tool in the control of influenza involves protective vaccinations. Since 1994, vaccinations against influenza have been among the recommended vaccinations in Poland. Health professionals belong to those professional groups for which vaccination is recommended due to epidemiological indications (Brydak, 2008).

\section{MATERIAL AND METHODS}

The study group consisted of medical students, i.e. those who either already had or in the future would have contact with patients. For the study, students of the following departments were selected from two universities (the University of Technology and $\mathrm{Hu}-$ manities in Radom, and the Medical University of Łódź): nursing (full-time courses and bridging courses), medical analysts, physiotherapy, medicine, and pharmacy. The study was conducted in the 2012/2013 season using a survey method based on the authors' questionnaire, containing information about the study, particulars (age, sex, place of residence, education) and 15 research questions. Of these, 13 were closed questions with the possibility of one response only, whereas the other two, apart from a choice of answers, allowed the respondents to write their own response. Participation in the survey was anonymous, voluntary, and unpaid. The results obtained have been elaborated using an Excel spreadsheet by defining the

e-mail: kaj12@poczta.fm 
percentage of each response to each of the questions in groups of questions examining the area of knowledge, opinions, and behaviours of respondents regarding influenza and influenza vaccination. Since the respondents did not provide answers to all the questions, the results expressed in percentages refer to the sum of all the responses to each question, and not to the number of respondents.

\section{RESULTS}

The study involved 470 people aged 19-43, of whom 423 respondents $(90 \%)$ were women and 47 respondents $(10 \%)$ men. The vast majority of respondents were urban residents $(80.6 \%)$; rural residents accounted for $19.4 \%$ of the respondents. The feedback estimated for each of the questions ranged from 55 to $75 \%$.

The basic knowledge of the respondents was examined by asking them about sources of information about influenza and vaccination, the time of year it is best to get vaccinated, the components of the virus which are the main element of influenza vaccines, complications from influenza, the average number of deaths worldwide from influenza, and its complications.

Among the available sources of information about influenza and vaccination the most popular among respondents were the mass media - $35.6 \%$, while only $20.6 \%$ rated them as understandable. Other sources of information included: medical staff $-27.9 \%$ of responses, scientific publications available on the internet $-22.3 \%$ of responses, family - $9.9 \%$ of responses, other sources - 4.3\% responses (ads in the clinic, friends). According to the respondents, the best time to get vaccinated is the beginning of autumn - 79.3\% of the responses, $9.6 \%$ of cases thought it did not matter, $4.1 \%$ - in winter, and $6.6 \%$ in spring. In terms of knowledge of the components of the virus present in the vaccine, as many as $55.5 \%$ of the respondents answered "I do not know". The other responses were: "parts of a virus" — $29.3 \%$ of responses, "glycoproteins" — $8.7 \%$ responses, "the whole virus" - 6.5\% of the responses. In terms of awareness of influenza-related complications, the result was $87.4 \%$ of the "yes" answers and $12.6 \%$ for "no", while only $19.1 \%$ of respondents indicated the correct number of deaths from influenza and its complications in the world in a given year.

Opinions on influenza and influenza vaccination were investigated by asking about the best ways of its prevention, and the appropriateness of vaccination of pregnant women and children over 6 months of age. In terms of ways to prevent influenza, respondents most often chose domestic procedures (garlic, onion, etc.) - $46.6 \%$ of responses, while only $20.6 \%$ of responses indicated vaccination; other possibilities: vitamins - 18.6\% of responses, analgesics and antipyretics $-8.7 \%$ of responses, and $6.8 \%$ of responses indicated that household remedies, medicines and vitamins only treat the symptoms. In terms of the appropriateness of vaccination of pregnant women, as many as $49.4 \%$ of the answers were "I have no opinion", $34.9 \%$ of respondents said that it was "a bad idea", and only $15.6 \%$ of the answers were: "a very good idea". Opinions regarding the appropriateness of vaccination of children over 6 years of age were as follows: $44.2 \%$ of responses — "I have no opinion", 27.1\% — "a bad idea", 28.6\% — "a very good idea".

Behaviours and the motivations were studied by asking about vaccination of the respondent in the past five

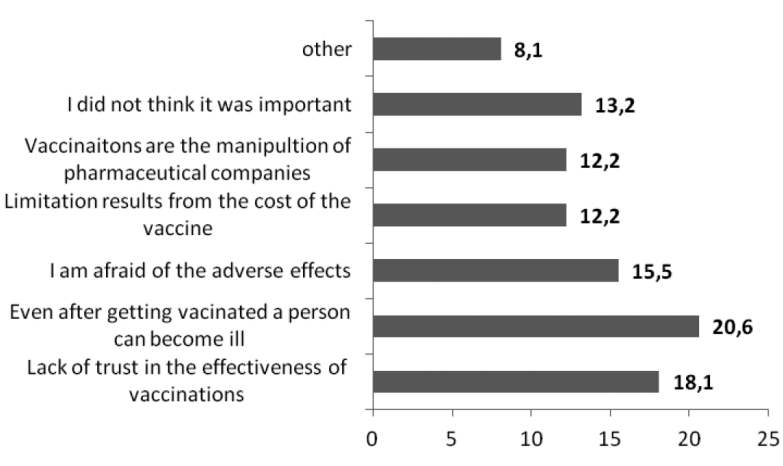

Figure 1. The reasons for non-vaccination against influenza in the last season (\% of responses)

seasons, about vaccination in the season to which the study was related, the reasons for non-vaccination of the respondent, if there is interest in free vaccinations, a tendency to encourage vaccination among family and friends, time and reason for which the respondent would be willing to get vaccinated.

In this respect, the following results were obtained. Regular vaccinations in the past 5 seasons were indicated by $5.8 \%$ responses, lack of vaccination in this period - $17.9 \%$ of responses, others: $47.5 \%$ - occasionally, and $28.8 \%$ - not regularly. Only $6.6 \%$ of the responses indicated vaccination during the season with which the study was concerned, while the remaining $93.4 \%$ of the responses indicated that the respondents were not vaccinated. Those who gave a negative answer to the question of vaccination during the season to which the study was related were asked an additional question designed to highlight the reasons for that decision. The results are shown in Fig. 1.

The most important reason was the belief that in spite of vaccination a person can still fall ill with influenza $(20.6 \%)$, which is associated with a lack of faith in the efficacy of vaccination $(18.2 \%)$. Quite a large group (15.5\% of respondents) indicated a fear of adverse reactions associated with vaccination. The "other" category (8.1\% of responses) included: "I do not feel the need to be vaccinated", and "I'm healthy". For some people the most important reason for not being vaccinated were financial constraints $(12.1 \%$ of responses), while in response to the question of whether free vaccine would encourage vaccination only $27.8 \%$ of the answers were "yes" and as many as 31\% "no". Most respondents, i.e. $41.3 \%$, had no opinion on the subject. When respondents were asked to encourage their family or friends, a substantial minority of respondents said "yes" (24.4\% of the responses), while the others said "no" (75.6\% of the responses). As far as the best time or reason to get vaccinated is concerned, as many as $43.8 \%$ of responses were "I have no opinion", 32.1\% of the responses were related to the fact of being in a risk group, and $24.1 \%$ replied that they would get vaccinated as soon as the vaccine was available on the market.

Our research confirmed unfavourable statistics regarding influenza immunization coverage among students of medical faculties. The reluctance of students to get vaccinated may in the future decrease even below the currently low immunization coverage in the total population and in the groups of increased risk. 


\section{DISCUSSION}

Findings from our research indicated that only $5.84 \%$ of students of medicine and other medical faculties received seasonal vaccination against influenza in 2012/2013. This rate is comparable to the total reported rate for health care personnel in Poland who received vaccination against influenza (Brydak et al., 2012; Woźniak-Kosek et al., 2014). A review study on the immunization coverage of influenza vaccine among health care personnel in selected countries demonstrated that $9-92 \%$ of them receive the vaccine. Immunization coverage in European countries is far lower than in the USA or Canada (Prematunge et al., 2012; Supranowicz et al., 2013).

According to our research, the most common source of knowledge about influenza and vaccination for the respondents (students) is the mass media, although only $20 \%$ evaluate the information obtained from there as understandable. This is very disturbing because respondents, due to their field of study, constitute a group with a higher level of medical knowledge than an average person. In the study group an important source of information comprises scientific publications available online, which is understandable because of its medical nature. Whereas, a small proportion of results indicating the family as the source of information suggests that it is the person being surveyed that should be the source of information for family members rather than vice versa. However, other studies indicated much higher percentages concerning the family as a source of information about vaccinations, but these studies related to a different group of respondents - the elderly, who at the same time may/may not be patients (Lukomska et al., 2009; Dymek-Skoczyńska et al., 2012). Although the vast majority of responses correctly indicated that autumn is the best time of year to get vaccinated, and that there is a possibility of complications from influenza, the knowledge concerning the main component of the vaccine and the mortality rate from influenza and its complications proved to be low. This may indicate a lack of interest among respondents in influenza and vaccinations as an effective method of preventing it, and that they do not search for information on this topic. It may also indicate that this group of respondents treats influenza as a rather mild disease, with cold-like symptoms which admittedly can lead to complications, that may prove fatal in many cases, but such complications are not associated with the disease,. This "underestimation" of the risk associated with the flu may be the result of, inter alia, the young age of the respondents and the consequent lack of a significant burden of chronic diseases, and thus a lower risk of experiencing complications in the case of their own illness. A positive assessment of their health condition (e.g. related to young age) is one of the important reasons why many people do not get vaccinated (Johnson et al., 2008; Lukomska et al., 2008). The fact that influenza is treated as one of the many diseases with cold-like symptoms may be reflected by opinions on the methods for how to prevent the flu: almost three quarters of respondents indicated household remedies as basic preventive measures (such as garlic or onions), vitamins, analgesics and antipyretics available without a prescription. The role in shaping such an opinion can be played, among others, by tradition - because people have long been trying to cope with various diseases using folk medicine. However, pharmaceutical companies play an important role in the mass media, where ads on various drugs are presented for the so-called cold, at the same time supplying information that they are also effective in the case of influenza. Only a few percent of responses point to the fact that these drugs can only alleviate the symptoms, whereas only one fifth of the responses indicated that vaccinations are effective preventive measures against influenza.

The relatively low approval for influenza vaccination expressed in the opinions is reflected in the behaviour of the respondents, resulting in very low immunization coverage among respondents, at approximately 5-6\%. This ratio is in fact slightly higher than for the general population, which in the $2012 / 2013$ season was $3.5 \%$, but it is still below the recommendations, especially when it comes to medical personnel (The National Program for influenza prevention, 2013). In the U.S., the influenza immunization coverage of medical personnel ranges from 20 to $80 \%$ (40-50\% on average) (Shefer et al., 2011). It is estimated that in Poland the proportion of vaccinated medical staff is $5-6 \%$ (which corresponds to the results of our study), and is slightly higher than vaccinations among the general population $3.7 \%$ in the 2012/13 epidemic season) (The National Program for influenza prevention, 2013). The influenza immunization coverage among medical staff depends on many factors, such as the occupation (doctors are vaccinated more often than nurses), specialization (most often paediatricians and family doctors are vaccinated, least frequently surgeons), age (most often vaccines are used by employees aged over 60), place of work (more frequently vaccines are used by hospital care employees than ambulatory staff (Nitsch-Osuch et al., 2013). Our research group was composed in a vast majority of women, who were students of nursing $(75 \%)$.

To conclude, it must be stated that the surveyed respondents showed a relatively low acceptance and an attitude of distrust of vaccination against influenza, which results in a very low immunization coverage, and does not provide sufficient encouragement for others for this method of prevention.

\section{CONCLUSIONS}

Knowledge about influenza and vaccination against influenza among medical students surveyed at the University of Technology and Humanities in Radom, and the Medical University of Łódź is relatively extensive, but reluctance to get vaccinated causes a significant dissonance between the acquired knowledge and prioritizing the protection of health, both students' own and of their future patients.

The immunization coverage in the group that was studied was low, whereas the cost of vaccination, although important, is not the main barrier to get of vaccinatated. The main reasons for not using vaccination as a method of prevention is the belief of the ineffectiveness of vaccination in the prevention of the disease, the fear of side effects of the vaccination, and a perception of the influenza problem as having too little importance. Vaccinations against influenza constitute activities which are not acceptable by people who already are or will become medical staff in the near future.

\section{REFERENCES}

Brydak LB (2008) Influenza - Pandemic Flu, a Myth Or a Real Threat? RYTM, Warsaw.

Brydak LB, Woźniak-Kosek A, Nitsch-Osuch A (2012) Influenza vaccines and vaccination in Poland. Med Sci Monit 18: 166-171. 
Dymek-Skoczyńska A, Stanisławska J, Drozd E, Talarska D (2012) Vaccination against influenza in the elderly - factors determining the decision of patients. Nowiny Lekarskie 81: 21-25.

Johnson D, Nichol K, Lipczyński K (2008) Barriers to adult immunization. Am J Med 121: 28-35.

Lukomska A, Janowska A (2007) Principles of prevention of influen$\mathrm{za}$ - their knowledge and use by patients. Probl Hig Epidemiol 88: $461-465$.

Łukomska A, Zielińska-Więczkowska H, Nowakowska I (2009) Factors determining the decision of patients to accept or resign from vaccinations. Probl Hig Epidemiol 90: 258-262.

Maszke M, Stasiak A, Kałucka S (2013) Similarities and differences in knowledge about the inoculation and level of vaccination against influenza among students in Lodz. Probl Med Rodz 15: 28-33.

Nitsch-Osuch A, Brydak LB (2013)Vaccination against influenza in medical personnel. Medycyna Pracy 64: 119-129

Prematunge C, Corace K, McCarthy A, Nair RC, Pugsley R, Garber G (2012) Factors influencing pandemic influenza of health workers: a systematic review. V accine 30: 4733-4743.
Shefer A, Atkinson W, Friedman C, Kuhar D, Mootrey G, Bialek S (2011) Immunization of health-care personnel. Recommendations of the advisory committee on immunization practices. MMWR Morb Mortal Wkly Rep 601-45.

Supranowicz P, Brydak LB (2013) Opinions of employees of the National Institute of Public Health-National Institute of Hygiene in Warsaw of Influenza Vaccination. Przegl Epidemiol 67: 667-674.

Woźniak-Kosek A, Mendrycka M, Saracen A, Kosek J, HallmannSzelińska E, Zielnik-Jurkiewicz B, Kempińska-Mirosławska B (2014) Vaccination status and perception of influenza vaccination in the Polish population. Advs Exp Med Biol DOI 10.1007/5584_2014_33 Springer International Publishing Switzerland 2014.

The National Program for influenza prevention (2013) www.opzg.pl. The Reports of influenza and suspected cases of influenza in Poland (2013) published by the NIPH-NIH www.pzh.gov.pl. 